\title{
Caracterización del Suicidio en el departamento de Sucre: Un análisis de las noticias publicadas en el Periódico El Meridiano de Sucre, Colombia
}

\section{Characterization of Suicide in the Department of Sucre: An Analysis of the News Published in EI Meridiano de Sucre Newspaper, Colombia}

\author{
José-Francisco Restrepo-Herrera \\ Corporación Universitaria del Caribe CECAR, Colombia \\ $\triangle$ jose.restrepo@cecar.edu.co \\ (iD) https://orcid.org/0000-0001-7280-6132 \\ Kelly Romero-Acosta* \\ Corporación Universitaria del Caribe CECAR, Colombia \\ $\triangle$ Kelly.romero@cecar.edu.co \\ (i) https://orcid.org/0000-0002-6568-1316 \\ Salomón Verhelst-Montenegro \\ Corporación Universitaria del Caribe CECAR, Colombia \\ $\triangle$ salomon.verhelst@cecar.edu.co \\ (ID) https://orcid.org/0000-0003-2206-4884
}

Recibido: $18-01-2019$

Aceptado: 04-03-2019

Publicado: 25-04-2019

\section{RESUMEN}

Objetivo. Analizar las noticias publicadas sobre suicidio consumado en el Periódico El Meridiano de Sucre del 2010 al 2016. Método. 1) la búsqueda se acotó a un tiempo específico (2010 - 2016); 2) se clasificó la información proporcionada por los artículos de periódico teniendo en cuenta: la fecha del acontecimiento, el lugar, la edad, el sexo, la modalidad y el motivo; 3) se introdujo toda la información al paquete estadístico Statistical Package for Social Science (SPSS v.21) para su análisis. Resultados. Se halló un aumento de las cifras del suicidio en los años 2015 y 2016 . Entre los motivos más frecuentes para cometer el acto suicida se encuentran: depresión, problemas familiares, problemas de salud y problemas económicos. Conclusiones. Tanto en Sincelejo como en la zona rural sucreña, la modalidad de acto suicida más frecuente en hombres y mujeres es el ahorcamiento, y el motivo más frecuente: "la depresión".

Palabras clave: Suicidio, Sucre, Sincelejo, Colombia

\section{ABSTRACT}

Objective. To analyze the news published in the newspaper El Meridiano de Sucre from 2010 to 2016, related to consummated suicide. Method. 1) the search was limited to a specific period of time: 2010 - 2016; 2) the information provided by the newspaper articles was classified taking into account: the date of the event, the place, the age, the sex, the modality and the reason; 3) all information was introduced for analysis into the Statistical Package for Social Science (SPSS v.21). Results. An increase in suicide rate 
was found between 2015 and 2016. Among the most frequent reasons for committing suicide are: depression, family, health and economic problems. Conclusions. Both in Sincelejo and in rural area, the most frequent type of suicidal act in men and women is hanging, and the most frequent reason: "depression".

Keywords: Suicide, Sucre, Sincelejo, Colombia

\section{RESUMO}

Objetivo. Analisar as notícias publicadas sobre o suicídio consumado no jornal El Meridiano, de Sucre, de 2010 a 2016. Método. 1) a busca foi limitada a um horário específico (2010 - 2016); 2) as informações fornecidas pelos artigos de jornal foram classificadas levando-se em conta: a data do evento, o local, a idade, o sexo, a modalidade e a razão; 3) todas as informações foram introduzidas no pacote estatístico Statistical Package for Social Science (SPSS v.21) para análise. Resultados. Um aumento nos números de suicídio foi encontrado em 2015 e 2016 . Entre os motivos mais frequentes para cometer o ato suicida estão: depressão, problemas familiares, problemas de saúde e problemas econômicos. Conclusões. Tanto em Sincelejo como em Sucreña rural, 0 tipo mais frequente do ato suicida em homens e mulheres está suspendendo, e a razão mais frequente: "a depressão".

Palavras-chave: Suicídio, Sucre, Sincelejo, Colombia

\section{INTRODUCCIÓN}

Judas, el que lo entregó, viendo que Jesús había sido condenado, lleno de remordimiento, devolvió las treinta monedas de plata a los sumos sacerdotes y a los ancianos, diciendo: «He pecado, entregando sangre inocente». Ellos respondieron: « ¿Qué nos importa? Es asunto tuyo». Entonces él, arrojando las monedas en el Templo, salió y se ahorcó (Mateo 27:3-10 La Biblia, El libro del pueblo de Dios (traducción argentina, 1990)).

La Organización Mundial de la Salud (OMS, 2014) en su informe Preventing Suicide distingue entre suicidio, intento de suicidio y comportamiento suicida. El primero, es el acto de matarse a sí mismo voluntariamente; el segundo, es un tipo de comportamiento suicida, sin consecuencias fatales, se incluye cualquier intento de dañarse a sí mismo: con o sin intención de matarse; el tercero, se refiere a todo comportamiento que esté orientado a dañarse a sí mismo y va desde los pensamientos hasta las acciones suicidas. El suicidio es un problema de salud pública reconocido a nivel mundial, que puede afectar a cualquier individuo, sin importar su sexo, edad, grupo étnico, estrato socioeconómico, raza y/o religión (Campo-Arias, 2015; Cardona, Médina-Pérez y Cardona, 2016; CastroRueda, Rangel-Martínez, Camacho, \& Rueda-Jaimes, 2010; González, Rodríguez y Aristizábal, 2010; Vásquez y Quijano, 2013). A nivel mundial las cifras de suicidio varían según el área geográfica; la Organización Mundial de la Salud (OMS, 2018) presenta la tasa de mortalidad por suicidio (por 100000 habitantes), deacuerdo con las diferentes regiones geográficas: África: 7.4; Américas: 9.8; Asia: 13.2; Europa: 15.4; Mediterraneo oriental: 3.9 y Pacífico Oeste: 10.2. Las tasas más altas se concentran en Europa y en el Pacífico Oeste. Colombia se encuentra un poco por debajo de la tasa de la región de las Américas, manteniendo un promedio en los últimos seis años de 7.4. Según el Instituto Nacional de Medicina Legal y Ciencias Forenses (2017), en Colombia ha habido un aumento del $11.30 \%$ en las cifras de suicidio anuales. La tasa de suicidio aumentó 
Restrepo et al - Caracterización del Suicidio en el departamento de Sucre.

52 puntos en $2017(n=2310$; tasa por 100000 habitantes: 5.20) con respecto a la tasa de 2016 ( $n=2571$; tasa por 100000 habitantes: 5.72). En este sentido, todos los esfuerzos que se hagan para disminuir las cifras de suicidio a nivel mundial y local son bienvenidas. En el caso de Colombia, el comportamiento suicida resulta ser un grave problema de salud pública (Redón-Quintero \& Rodríguez-Gómez, 2016) y su estudio debe ser prioritario, más aún teniendo en cuenta la situación de precariedad en la que viven muchas familias colombianas, causada directa o indirectamente por el conflicto armado.

Ahora bien, algunos investigadores y clínicos consideran que, para prevenir el comportamiento suicida en todos los grupos de edad, es necesario tener en claro los factores de riesgo y las fortalezas, con el fin de mejorar las estrategias de atención (ColeKing \& Platt, 2017). Los factores de riesgo y protectores varían según el grupo de edad, el género y el lugar de procedencia, lo cual aumenta la complejidad del comportamiento suicida. A continuación se detallarán algunos factores de riesgo que se han relacionado con la presencia de comportamiento suicida especialmente en Colombia.

Comenzaremos, pues, con el factor económico y el familiar. Campo-Arias (2015) señala que existe una correlación positiva entre desigualdad económica y tasa de suicidio en Colombia. Este dato lo corrobora el estudio realizado por Valencia, Campo-Cabal, Borrero, García, \& Patiño (2011) quienes hallaron que el intento de suicidio se incrementa en personas de estratos socioeconómicos bajos. También, Castro-Díaz et al., (2013) hallaron que la ideación suicida se relaciona con personas que tengan bajos ingresos y con haber sufrido una crisis financiera en los últimos dos años. En ocasiones, los problemas económicos generan problemas en las relaciones de los miembros de la familia. CastroRueda et al., (2010) y González et al., (2013) encontraron que los problemas familiares y de pareja se relacionan en alto grado con el comportamiento suicida, sin embargo, no tiene en cuenta la variable de ingresos económicos en su análisis.

Las zonas rurales y urbanas tienen sus propias características relacionadas con el comportamiento suicida. En un estudio desarrollado en Antioquía, se hallaron diferencias significativas entre los suicidios sucedidos en áreas rurales y urbanas (García, Montoya, Jaramillo, López, \& Palacio, 2011). El envenenamiento con pesticidas es significativamente más frecuente en zonas rurales y el "vivir solo" es la característica más frecuente en los suicidios llevados a cabo en zonas urbanas.

Por otra parte, investigaciones anteriores afirman que el género puede relacionarse con un riesgo mayor o menor del comportamiento suicida. Varios estudios han hallado una incidencia del comportamiento suicida mayor en hombres que en mujeres (Cardona, Médina-Pérez, \& Cardona, 2016; Castro-Díaz, Gómez-Restrepo, Gil, Uribe, \& Pinto, 2013; González, et al., 2010; Muñoz \& Gutierrez, 2010; Peña, Ortiz, \& Gutierrez, 2009). En la encuesta nacional de salud mental realizada en el 2015 , el $5.5 \%$ de los varones participantes y el $7.6 \%$ de las mujeres pensaron en suicidare alguna vez en su vida (Arenas, Gómez-Restrepo, \& Rondón, 2016). González y sus colegas (2010), encontraron que los varones tienden a cometer el acto suicida con arma de fuego, generalmente viven solos y consumen alcohol al momento del acto; mientras que las mujeres, tienden a suicidarse en casa y tienden a dejar una nota. Por su parte, RuedaJaimes y colaboradores (2011) hallaron que el uso y el abuso de sustancias y el consumo de alcohol se asociaron a los hombres con comportamiento suicida.

Con respecto al factor etario, en 2016, Cardona y sus colegas, realizaron una investigación descriptiva, teniendo en cuenta los certificados de defunción de personas fallecidas por suicidio en Colombia; éstos hallaron un total de 24.882 suicidios consumados desde el 
año 2000 al 2010, cuya edad promedio fue de 34,5. El suicidio presenta rasgos específicos para cada etapa del ciclo vital o grupo de edad de las personas que lo llevan acabo (Valencia, Campo-Cabal, Borrero, García, \& Patiño, 2011; Velázquez, 2013). Vásquez y Quijano (2013) realizaron una investigación sobre intento de suicidio infanto-juvenil en Colombia; ellos concluyeron que existe mayor riesgo de realizar intentos suicidas en la adolescencia (Muñoz \& Gutierrez, 2010), hecho que también halló Rueda-Jaimes, et al., (2010) y González, et al., (2013). En la adolescencia, el acto suicida se hace generalmente con medicamentos y frecuentemente tiene comorbilidad psiquiátrica, prevaleciendo los trastornos internalizados como la ansiedad y la depresión (Vásquez \& Quijano, 2013). En efecto, en 2012, Pérez-Olmos, et al., hallaron una relación entre comportamiento suicida y la comorbilidad psiquiátrica, sobre todo con depresión, además, otros factores asociados fueron: el estrato-socio-económico, ser mujer, la disfunción familiar severa y el haber sufrido de abuso físico.

A pesar de que el suicidio en el anciano tiene pocos estudios realizados en Colombia (Velázquez, 2013), hoy por hoy se sabe que los suicidas de este grupo de edad (mayores de 60 años) son en su mayoría hombres y que entre las principales causas de suicidio se encuentran: padecimientos de enfermedades crónicas y discapacitantes; en el caso de las mujeres adultas mayores, las principales causas de suicidio se asocian a la presencia de algún trastornos mental o a la pérdida de un ser querido (Ordoñez, Franco, \& Gozález, 2013).

En efecto, también se han hallado características específicas del comportamiento suicida relacionadas con el factor de la salud mental. Por ejemplo, se ha relacionado el comportamiento suicida con la presencia de trastornos mentales, sobre todo con depresión y con abuso de sustancias, tales como alcohol y diferentes tipos de drogas (Castro-Rueda, et. al. 2010; Rodriguez, Rodríguez, Gempeler, \& Garzón, 2013; Muñoz \& Gutierrez, 2010; González, Castilla, Retamoza, \& Bolaños, 2013; Muñoz, Bernal, Guarín, \& Arévalo, 2015; Valencia, et al., 2011; Gómez-Restrepo, Bohórquez, Tamayo, Rodón, \& Medina, 2015; ; Castro-Díaz et al., 2013). Gómez-Restrepo, et al., (2016) indican que entre los factores asociados con la ideación suicida se encuentran la percepción de la salud mental como regular o mala y el diagnóstico de depresión doble. También, parece que los medios de comunicación podrían influir en la aparición de las conductas suicidas, los jóvenes o personas que sufren trastorno de depresión mayor, podrían ser ponencialmente vulnerables a imitar conductas suicidas de otros (Muñoz \& Sánchez, 2013). La soledad y el dolor psíquico se relacionarían con la desesperanza, el pesimismo y la desmotivación, es realmente esencial tener en cuenta la valoración subjetiva que los individuos, con este tipo de comportamientos, hacen de la depresión y de las situaciones estresantes, porque esto puede evitar la consumación del suicidio en el futuro (RedónQuintero \& Rodríguez-Gómez, 2016).

El comportamiento suicida no sólo se encuentra supeditado a la constelación sintomática del trastorno mental en sí, sino, también, al estigma que produce tener ese trastorno mental. Campo-Arias \& Herazo (2015) señalan que el complejo estigma-discriminación asociado al trastorno mental impacta de manera negativa la calidad de vida de las personas, lo que se convierte en un factor de riesgo para la presentación de conductas suicidas. Este impacto puede ocurrir de dos maneras, por una parte, el estigma percibido puede configurarse como barrera de acceso a los servicios de salud mental, por otra parte, los comportamientos autolesivos reducen la autoestima e incrementan el estrés.

Como se ha podido ver hasta ahora, hay características específicas del comportamiento suicida a nivel nacional. Éste se relaciona con la presencia de problemas familiares, el género, el grupo de edad, el estrato socio-económico, la presencia de trastornos mentales 
previos, entre otros. Vale la pena destacar que las cifras de suicidio también varían según el lugar geográfico. Tal y como vimos anteriormente con las cifras mundiales, Europa y el Oeste pacífico son mundialmente las regiones con tasas de suicidio más altas para el año 2017. En Colombia sucede igual, veamos el comportamiento de la tasa de suicidios en la región caribe. Según el informe emitido anualmente por el Instituto de Medicina Legal desde 2010 hasta 2016, el promedio de la tasa de suicidios a nivel nacional se ha mantenido en 4.41. Especificamente en la región caribe, el departamento de César y Sucre son los que presentan, en promedio, las tasas más altas de la región (4.64 y 4.09, respectivamente). Esta información se encuentra más detallada en el gráfico 1.

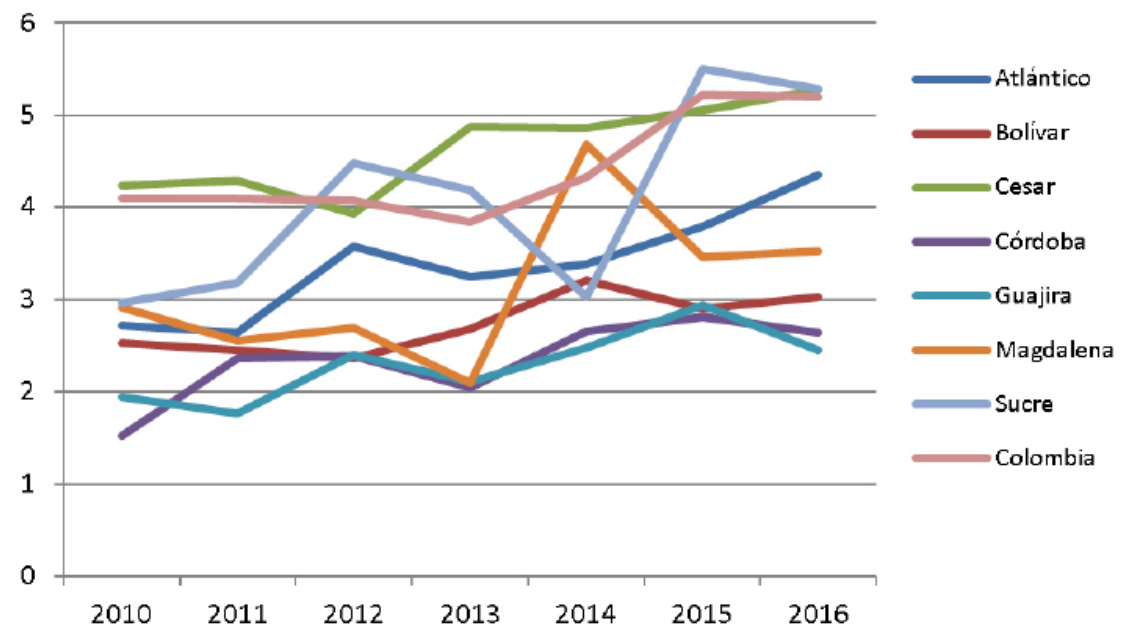

Fuente: Instituto Nacional de Medicina legal (2010 - 2016). Elaboración propia.

Con respecto a las capitales de los departamentos de la región caribe, Sincelejo $(5,50)$, es el que presenta en promedio la tasa más alta de 2010 a 2016, seguida por Valledupar $(4,81)$, Monteria $(4,46)$ y Santa Marta $(4,07)$.

Son muchas las razones por las cuales la tasa de suicidios varía según el espacio geográfico, sobre todo, por las características relacionadas con el grupo cultural, incluso por acontecimientos vitales estresantes que pueden vivirse a nivel colectivo, como un desastre natural o una situación violenta. Al respecto, Gómez-Restrepo, et al., (2016) señalan que los municipios violentos tienden a presentar más prevalencia de tratorno de ansiedad, depresión y trastorno de estrés postraumático que otros municipios cuya vivencia del conflicto fue menor. Ciertamente cuando el contexto es adverso, puede verse incrementado el riesgo de padecer una psicopatología (Boldrini \& Mann, 2015). Esto es muy importante porque como se ha señalado anteriormente, la presencia de trastornos mentales aumenta la probabilidad de ideación suicida, intento suicida y/o suicidio. Estudios anteriores han encontrado que los hechos traumáticos vividos en el marco del conflicto armado se relacionan con la presencia de trastornos mentales, sobre todo con ansiedad y/o depresión (Alejo, 2005; Botelho \& Conde, 2011; Echenique, Medina, Medina, \& Ramírez, 2008; Londoño, Muñiz, \& Correa, 2005; Palacio, Mandariaga, \& Sabatier, 2001; Hewitt, y otros, 2016). En Sucre se destacan los estudios de Echenique et al., (2008) y Ramírez et al (2017), quienes encuentran niveles elevados de psicopatología en personas afectadas por el conflicto armado.

El departamento de Sucre es uno de los departamentos más afectados por el conflicto armado. A fecha de junio de 2018, el Registro Único de Víctimas (RUV) indica que las víctimas registradas historicamente en todo el país ascienden a 8'695.848 (RUV, 2018). En Sucre, el número exacto de víctimas asciende a: 291.670. Se sabe que el 
conflicto colombiano trae consigo una serie de acontecimientos estresantes que podrían relacionarse con la presencia de trastornos mentales, sobre todo, de tipo afectivo y ansioso (Ramírez-Giraldo, et al., 2017), estos trastornos psicopatológicos guardan estrecha relación con el comportamiento suicida, sobre todo, la depresión. En Colombia, la ley 1616 de Salud Mental, en su artículo 8, indica que entre las acciones de promoción en salud mental que el Ministerio de Salud y Protección Social priorizará se encuentra la prevención del suicidio (Ley 1616, 2013). En este sentido, todos los esfuerzos que se realicen con la intención de movilizar programas de promoción de la salud mental y prevención del trastorno mental, son vitales para disminuir las cifras de suicidio.

En la actualidad no se tienen cifras exactas del comportamiento suicida en el departamento de Sucre, en este estudio se pretende dar un primer esbozo de la situación, a través de uno de los medios de comunicación más confiables del departamento: El periódico El Meridiano de Sucre. El objetivo de este estudio es analizar las noticias publicadas sobre suicidio consumado en este Periódico del 2010 al 2016 .

\section{MÉTODO}

Procedimiento. Este estudio tiene el permiso del comité de Ética de la Corporación Universitaria del Caribe CECAR. En primer lugar, se escogió el periódico más influeyente en la región: El Meridiano de Sucre, se acotó a un tiempo específico -es decir desde el año 2010 hasta el 2016-, se rastrearon todos los acontecimientos de suicidio, se clasificó la información proporcionada por los artículos de períodico teniendo en cuenta: la fecha del acontecimiento, el lugar, la edad, el sexo, la modalidad y el motivo. Una vez obtenida esta información, se introdujo en el paquete estadístico Statistical Package for Social Science (SPSS v.21) para su análisis descriptivo. Posteriormente, se contrastaron los resultados con investigaciones realizadas anteriormente sobre el tema en el país.

Análisis estadístico. Para el análisis estadístico se utilizó el Statistical Package for Social Scince (SPSS) v. 21, los estadísticos que se usaron fueron principalmente descriptivos: sobre todo frecuencias y porcentajes.

\section{RESULTADOS}

A continuación se presentan los resultados del análisis de las noticias publicadas en el periódico El Meridiano desde 2010 hasta 2016. En líneas generales, la cifra de hombres que cometieron acto suicida fue más alta que la de las mujeres $(n=151,84,4 \% ; n=28$, $15,6 \%$, respectivamente); y la media de edad fue de 46,46 (DS $=23,69)$; especificamente para las mujeres la media de edad fue de $31,9(D S=19,8)$ y para los hombres 49,2 $(\mathrm{DS}=23,4)$.

Cifras de suicidio por año y mes. El análisis bibliográfico sugiere que hay un aumento de las cifras de suicidios en los años 2015 y 2016, duplicando las cifras de 2010 y 2011. El gráfico 1 muestra de forma más detallada esta información. Con respecto a los meses con más frecuencia de suicidio según el periódico utilizado, se tiene que abril y agosto son los meses con más cifras de suicidio ( $n=20$ y 19 respectivamente) y noviembre y febrero con menos ( $n=11$ y 9 , respectivamente).

Cifras de suicidio por municipio. En la ciudad de Sincelejo se han presentado el $42 \%$ de los casos de suicidio de Sucre, seguido por Corozal y San Marcos. Los municipios de San Roque, El Roble y Guaranda, fueron los que presentaron las menores cifras de suicidio en los últimos siete años (una persona). 
Motivo de los actos suicidas. Lastimosamente, $41.3 \%$ de las noticias no registraron el motivo específico del suicidio. Sin embargo, entre los motivos más frecuentes tenemos: depresión, problemas familiares, problemas de salud y problemas económicos. Toda esta información se puede ver de manera más detallada en la tabla 1.

Tabla 1. Motivos de acto suicida

\begin{tabular}{lcc}
\hline & Frecuencia & Porcentaje \\
\hline Desconocido & 74 & 41,3 \\
Depresión & 23 & 12,8 \\
Problemas familiares & 18 & 10,1 \\
Problemas de salud & 16 & 8,9 \\
Problemas económicos (deudas) & 11 & 6,1 \\
Problemas sentimentales & 8 & 4,5 \\
Problemas psiquiátricos & 7 & 3,9 \\
Depresión por problemas de salud & 6 & 3,4 \\
Falta de empleo & 3 & 1,7 \\
Muerte familiar & 2 & 1,1 \\
Otros & 7 & 3,9 \\
Consumo de sustancias & 1 &, 6 \\
Separación de pareja & 1 &, 6 \\
Acabar problemas que le agobian & 1 &, 6 \\
Problemas con vecinos & 1 & .6 \\
\hline
\end{tabular}

Modalidad de acto suicida. La modalidad más frecuente fue el ahorcamiento, seguida por arma de fuego y envenenamiento. Tanto para Sincelejo como para la zona rural sucreña, la modalidad de acto suicida más frecuente es el ahorcamiento, sin embargo, en la capital, la segunda modalidad de acto suicida es el arma de fuego, seguida por envenenamiento. Esta información se puede ver de manera más detallada en la tabla 2.

Tabla 2. Tipos de actos suicidas

\begin{tabular}{|c|c|c|c|}
\hline & & Frecuencia & Porcentaje \\
\hline \multirow{9}{*}{ 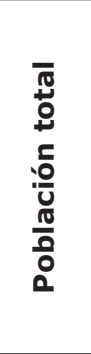 } & Ahorcamiento & 124 & 76,1 \\
\hline & Envenenamiento & 15 & 10,26 \\
\hline & Arma de fuego & 16 & 9,37 \\
\hline & Por incineración & 2 & 1,2 \\
\hline & Lanzamiento & 2 & 1.2 \\
\hline & Se prendió fuego & 1 & 6 \\
\hline & Degollamiento & 1 & 6 \\
\hline & Arma blanca en el estómago & 1 & 6 \\
\hline & Atropellado & 1 & 6 \\
\hline \multirow{5}{*}{ 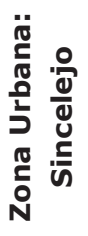 } & Ahorcamiento & 50 & 73,5 \\
\hline & Arma de fuego & 9 & 13,2 \\
\hline & Envenenamiento & 6 & 8,8 \\
\hline & Lanzamiento & 2 & 3.0 \\
\hline & Atropellado & 1 & 1,5 \\
\hline \multirow{7}{*}{ 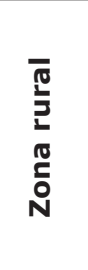 } & Ahorcamiento & 74 & 77,9 \\
\hline & Envenenamiento & 9 & 9,5 \\
\hline & Arma de fuego & 7 & 7,4 \\
\hline & Se prendió fuego & 1 & 1,1 \\
\hline & Degollamiento & 1 & 1,1 \\
\hline & Arma blanca en el estómago & 1 & 1,1 \\
\hline & Por incineración & 2 & 2,1 \\
\hline
\end{tabular}


Motivo y Modalidad del suicidio: Una comparación entre hombres y mujeres. Tanto para mujeres como para hombres las modalidades de suicidio más frecuentes son el ahorcamiento y el envenenamiento. Teniendo en cuenta que la cifra de los hombres fue mucho mayor, en comparación con las mujeres, éstos presentan modalidades de suicidio más variadas, tales como el uso de arma de fuego o el lanzamiento al vacío. Toda esta información se encuentra mucho más explicada en la tabla 3.

Tabla 3. Modalidad del suicidio por sexo

\begin{tabular}{clcc}
\hline \multicolumn{1}{c}{ Sexo } & & Frecuencia & Porcentaje \\
\hline Femenino & Envenenamiento & 7 & 29,2 \\
& Ahorcamiento & 16 & 66,7 \\
& Se prendió fuego & 1 & 4,2 \\
\hline \multirow{2}{*}{ Masculino } & Envenenamiento & 8 & 5,8 \\
& Ahorcamiento & 108 & 77,7 \\
& Arma de fuego & 16 & 11,5 \\
& Lanzamiento & 2 & 1.4 \\
& Degollamiento & 1 & 7 \\
& Arma blanca & 1 & 7 \\
& Atropellado & 1 & 7 \\
& Por incineración & 2 & 1,4 \\
\hline
\end{tabular}

Por otro lado, los motivos del acto suicida varían entre hombres y mujeres. Las mujeres presentan como motivos del acto suicida más frecuentes: la depresión, los problemas económicos y sentimientales. Los hombres, por su parte, presentan como móviles del acto suicida: la depresión, los problemas de salud y familiares.

Tabla 4. Motivos del acto suicida

\begin{tabular}{clcc}
\hline Sexo & & Frecuencia & Porcentaje \\
\hline Femenino & Desconocido & 9 & 32,1 \\
& Depresión & 6 & 21,4 \\
& Problemas psiquiátricos & 1 & 3,6 \\
& Problemas de salud & 1 & 3,6 \\
& Problemas económicos (deudas) & 2 & 7,1 \\
& Problemas sentimentales & 2 & 7,1 \\
& Problemas familiares & 3 & 10,7 \\
& Acabar problemas que le agobian & 1 & 3,6 \\
& Otros & 3 & 10,7 \\
\hline Masculino & Desconocido & 65 & 43,0 \\
& Consumo de sustancias & 1 &, 7 \\
& Depresión & 17 & 11,3 \\
& Problemas psiquiátricos & 6,0 \\
& Separación de pareja & 6 & 7 \\
& Problemas de salud & 1 & 9,9 \\
& Problemas económicos (deudas) & 15 & 6,0 \\
& Problemas sentimentales & 9 & 4,0 \\
& Falta de empleo & 6 & 2,0 \\
Problemas familiares & 3 & 9,9 \\
& Muerte familiar & 15 & 1,3 \\
Otro intento suicidio consumado & 2 &, 7 \\
Otros & 1 & 2,0 \\
Problemas con vecinos & 3 & 4,0 \\
Depresión por problemas de salud & 1 & 7 \\
\hline
\end{tabular}


Restrepo et al - Caracterización del Suicidio en el departamento de Sucre.

\section{DISCUSIÓN}

El objetivo de este estudio es analizar las noticias publicadas sobre suicidio consumado en el Periódico El Meridiano de Sucre del 2010 al 2016. Debido a que esta investigación es un análisis documental de las noticias presentadas en el susodicho perídico, no contamos con información precisa sobre la raza, la ocupación, el estado civil, entre otras cosas. Sin embargo, realizamos el análisis con la información suministrada por las noticias, en las que generalmente se encuentra el sexo, la edad, el motivo y el modo como la persona se mató. A partir de estos datos, se hace un sencillo, pero no por ello menos importante análisis del comportamiento suicida en Sucre.

A partir de esta investigación ahora se confirma que existe un aumento de las cifras del suicidio en Sucre en los años 2015 y 2016, superando en gran medida las cifras de 2010 y 2011. Estos datos son similares a los publicados por el Instituto Nacional de Medicina Legal y Ciencias Forenses de 2010 a 2016, el cual indica que en 2010 y 2011 la tasa de suicidios en Sucre fue de 2,96 y 3,18 respectivamente, mientras que en 2015 y 2016 fue de 5,5 y 5,29 respectivamente. En 2017 la tasa de suicidio se mantuvo en 5,10. En nuestro estudio, Sincelejo tuvo la mayor presencia de casos de suicidio de todo el departamento. Esto también lo halló el Instituto Nacional de Medicina Legal y Ciencias Forenses en sus informes de 2010 a 2016; en promedio Sincelejo mantuvo una tasa de 5,50, muy por encima de otras capitales del caribe como: Barranquilla $(3,92)$, Cartagena $(3,47)$, Valledupar $(4,81)$, Montería $(4,46)$, Riohacha $(3,00)$ y Santa Marta $(4,07)$.

En línea con estudios anteriores, en nuestro estudio, la cifra de hombres que cometieron acto suicida fue más alta que la de las mujeres (Arenas, Gómez-Restrepo, \& Rondón, 2016; Cardona, Médina-Pérez, \& Cardona, 2016; Castro-Díaz, Gómez-Restrepo, Gil, Uribe, \& Pinto, 2013; González, et al., 2010; Muñoz \& Gutierrez, 2010). Tanto para mujeres como para hombres las modalidades de suicidio más frecuentes fueron el ahorcamiento y el envenenamiento. No obstante, los hombres presentaron modalidades de suicidio más variadas, tales como el uso de arma de fuego o el lanzamiento al vacío. Estos hallazgos son similares a los de González y sus colegas (2010), quienes encontraron que los varones tienden a cometer el acto suicida con arma de fuego, generalmente viven solos y consumen alcohol al momento del acto; mientras que las mujeres, tienden a suicidarse en casa y tienden a dejar una nota. Las mujeres de Sucre, tienden a suicidarse a través del envenenamiento o del ahorcamiento. Según la OMS (2014) estos métodos son los más utilizados a nivel mundial, sin embargo, se sabe que pueden variar dependiendo del grupo poblacional, de la edad y/o del género. García, et al., (2011) señalan que el envenenamiento con pesticidas es significativamente más frecuente en zonas rurales. En Sucre, tanto en zonas rurales como en zona urbana el envenenamiento es el medio más utilizado para cometer el acto suicida, aunque, en Sincelejo, la capital, el uso de arma de fuego y el lanzamiento (de edificios/en carretera) es mucho mayor comparatibamente. Esto puede deberse a que a pesar de que Sincelejo es la urbe del departamento de Sucre, su infraestructura y la vida de sus ciudadanos es más parecida a pueblos grandes como su vecino Corozal.

Con respecto a las causas del suicidio se halló que la depresión, los problemas familiares, los problemas de salud y los problemas económicos fueron las causas más frecuentes para cometer actos suicidas entre los ciudadanos.

En estudios anteriores se ha relacionado el comportamiento suicida con la presencia de trastornos mentales, sobre todo con depresión (Castro-Rueda, et. al. 2010; Muñoz \& Gutierrez, 2010; Valencia, et al., 2011; Gómez-Restrepo, et al., 2015; Castro-Díaz et al., 2013). En nuestro estudio, algunas noticias señalaban que la depresión se 
debía a problemas de salud tales como: triglicéridos altos, cáncer, entre otros. Estos resultados son similares a los encontrados por investigaciones anteriores, en las cuales las principales causas de suicidio son los padecimientos de enfermedades crónicas y discapacitantes (Velázquez, 2013; Ordoñez et al., 2013). Esto es importante tenerlo en cuenta en los programas de atención a enfermedades crónicas tales como cáncer, cardiopatías, discapacidad debida a accidentes de tráfico, entre otras. También, es de vital importancia tenerlo en cuenta, sobre todo, en el adulto mayor, quien además de problemas de salud puede presentar problemas económicos, y esa fue otra de las causas más frecuentes para cometer el acto suicida en el departamento de Sucre.

Según Campo-Arias (2015) existe una correlación positiva entre desigualdad económica y tasa de suicidio en Colombia. Nosotros no podemos saber este hecho con exactitud, porque no tenemos los datos concretos sobre estatus socioeconómico, sin embargo, en varios casos el factor económico juega un papel importante en la vida de los individuos que eligen no continuar con sus vidas. Otros autores también señalan que el suicidio se incrementa en personas de estratos socioeconómicos bajos y con haber sufrido una crisis económica en los últimos dos años (Valencia, et al., 2011; Castro-Díaz et al., 2013).

También, entre las causas del comportamiento suicida se encontraron los problemas de pareja, si bien no fueron los más frecuentes, es preciso señalar que en el departamento de Nariño, fue la causa más frecuente de los suicidios (Peña et al., 2009). Esto nos da una idea de lo importante de hacer este tipo de estudios en diferentes regiones del país, pues las características varían según el espacio geográfico.

A partir de este estudio, ahora sabemos que el departamento de Sucre ha aumentado las tasas de suicidio desde el 2010 hasta el 2016. Lastimosamente no podemos sacar conclusiones definitivas sobre el motivo por el cual se ha generado este aumento en las cifras de suicidio de este lugar geográfico del país, sobre todo, en la capital: Sincelejo. Boldrini y Mann, (2015) señalan que cuando un país experimenta un cambio dramático, las cifras de suicidio pueden variar, y ponen como ejemplo la situación de algunos estados del este de Europa, los cuales presenciaron un cambio brusco en las cifras de suicidio cuando hubo la transición del comunismo a la democracia. No sabemos si el conflicto armado puede ser un motivo para que las cifras de suicidio en este punto geográfico del país hayan aumentado significativamente, valdría la pena investigarlo. Lo que sí es seguro es que en estudios anteriores realizados en el departamento, se ha encontrado que las víctimas del conflicto han visto afectada su salud mental (RamírezGiraldo, et al., 2017; Santis, Romero-Acosta, Sahagún-Navarro, \& Sánchez-Vergara, 2017; Sahagún et al., 2017) y la literatura apunta a que algunos trastornos mentales se asocian directamente con el comportamiento suicida (Carretta, Burguess, \& Welner, 2015; Trout, Hernández, \& Liu, 2017; Spino, Kameg, Cline, Terhorst, \& Mitchell, 2016).

Esta investigación más que respuestas, nos plantea interesantes interrogantes, sobre todo, relacionados con la sociedad sucreña. Todo este análisis nos genera una importante pregunta: ¿Qué está sucediendo en Sucre para que las cifras de suicidio de los habitantes se hayan duplicado en los últimos años? Se requieren estudios más profundos sobre el tema, que amplien los motivos especifícos de estas personas que deciden quitarse la vida, de hecho, sería recomendable llevar a cabo estudios con personas de todas las edades que visiten los servicios de salud por causa de ideación suicida o de intento de suicidio. Esto ayudaría a saber con presición qué sucede en la vida de estas personas incluso antes de que cometan el acto suicida o reincidan, para así poder plantear intervenciones más efectivas que ayuden a disminuir las cifras de suicidio en el departamento de Sucre. 
Restrepo et al - Caracterización del Suicidio en el departamento de Sucre.

\section{REFERENCIAS}

Alejo, E. (2005). Aplicación del modelo de estrés postraumático en el estudio del impacto de la violencia sobre la salud mental en población desplazada. MebUNAB, 8(1), 23-28.

Arenas, A., Gómez-Restrepo, C., \& Rondón, M. (2016). Factores asociados a la conducta suicida en Colombia. Revista colombiana de psiquiatría, 45(1), 68 - 75.

Boldrini, M., \& Mann, J. (2015). Depression and Suicide. En Neurobiology of Brain Disorder (págs. 709 - 729). NewYork: Columbia University Press.

Botelho, O., \& Conde, C. (2011). Memoria emocional y trastorno por estrés postraumático en el contexto del desplazamiento. Revista Colombiana de Psiquiatría, 40(3), 457-469.

Buendía, J., \& Mira , J. (1993). Estrés y psicopatología. Madrid. España: Pirámide.

Campo-Arias, A., \& Herazo, E. (2015). El complejo estigma-discriminación asociado al trastorno mental como factor de riesgo de suicidio. Revista colombiana de psiquiatría, 44(4), $243-250$.

Campo-Arias, E. (2015). Asociación entre desigualdad y tasa de suicidio en Colombia (1994 2013). Revista Colombiana de Psiquiatría, 44(1), 243 - 250.

Cardona, D., Médina-Pérez, A., \& Cardona, D. (2016). Caracterización del suicidio en Colombia, 2000 - 2010. Revista Colombiana de Psiquiátria, 45(3), 170 - 177.

Carretta, C., Burguess, A., \& Welner, M. (2015). Gaps in Crisis Mental Health: Suicide and Homicide-Suicide. Arch of Psych Nurs, 29(5), 339 - 345.

Castillo-Santis, S., Romero-Acosta, K., Sahagún-Navarro, M., Sánchez-Vergara, A.C. (2017). Evaluación de la salud mental de personas mayores, víctimas del conflicto armado, atendidas en el Centro de Orientación Sociojurídica de Sincelejo en los años 2012-2013. Búsqueda, 4(18), 62-71. DOI: https://doi.org/10.21892/01239813.338

Castro-Díaz, S., Gómez-Restrepo, C., Gil, F., Uribe, M., \& Pinto, D. (2013). Factores de riesgo para ideación suicida en pacientes con trastorno depresivo en Colombia. Revista Colombiana de Psiquiatría, 43(1), 27 - 35.

Castro-Rueda, A., Rangel-Martínez, A., Camacho, P., \& Rueda-Jaimes, G. (2010). Factores de riesgo y protectores para intento suicida en adultos colombianos. Revista Colombiana de Psiquiátria, 39(4), 705 - 715.

Cole-King, A., \& Platt, S. (2017). Suicide prevention for physicians: identification, intevention and mitigation of risk. Medicine, 45(3), 131 - 134.

Durkheim, E. (1998). El suicidio. Madrid: Akal.

Echenique, C., Medina, L., Medina, A., \& Ramírez, A. (2008). Prevalencia del trastorno por estrés postraumático en población desplazada por violencia, en proceso de restablecimiento. Psicología desde el Caribe, 21, 122 - 135. 
García, J., Montoya, G., Jaramillo, C., López, M., \& Palacio, C. (2011). Características de los suicidios de áreas rurales y urbanas de Antioquía, Colombia. Revista Colombiana de Psiquiatría, 40(2), 199-214.

Garciandia, J. (2013). Familia, suicidio y duelo. Revista colombiana de psiquiatría, 43(Supplement 1), 71 - 79.

Gómez-Restrepo, C., Bohórquez, A., Tamayo, N., Rodón, M., \& Medina, M. (2015). Trastornos depresivos y de ansiedad y factores asociados en la población de adolescentes colombianos. Revista Colombiana de Psiquiatría, 45(1), 50 - 57.

Gómez-Restrepo, C., Bohórquez, A., Tamayo, N., Rondón, M., \& Medina, M. (2016). Trastornos depresivos y de ansiedad y factores asociado en la población adulta colombiana, Encuenta Nacional de Salud Mental 2015. Revista Colombiana de Psiquiatría, 45(1), 58 - 67.

Gómez-Restrepo, C., Tamayo-Martínez, N., Buitrago, G., Guarnizo-Herreño, C., \& Rincón, J. (2016). Violencia por conflicto armado y prevalencias de trastornos del afecto, ansiedad y problemas mentales en la población adulta colombiana. Revista Colombiana de Psiquiatría, 45(1), 147 - 153.

González, A., Castilla, A., Retamoza, N., \& Bolaños, G. (2013). Factores de riesgo relacionados con los intentos de suicidio en Cartagena, Colombia. Acta psiquiátrica y psicológica de América Latina, 59(4), 240 - 252.

González, A., Rodríguez, Á., \& Aristizábal, A. (2010). Suicidioy género en Antioquia (Colombia): Estudio de autopsia psicológica. Revista Colombiana e psiquiatría, 39(2), $251-267$.

Hewitt, N., Juarez, F., Parada , A., Guerrero, J., Romero, Y., Salgado, A., \& Vargas, M. (2016). Afectaciones Psicológicas, Estrategias de afrontamiento y niveles de resiliencia de adultos expuestos al conflicto armado en Colombia. Revista Colombiana de Psicología, 25(1), 125 - 140.

Instituto Nacional de Medicina Legal y Ciencias Forenses. (2010). Forensis. Datos para la vida. Bogotá: Imprenta Nacional de Colombia.

Instituto Nacional de Medicina Legal y Ciencias Forenses. (2011). Forensis. Datos para la vida. Bogotá: Imprenta Nacional de Colombia.

Instituto Nacional de Medicina legal y Ciencias Forenses. (2012). Foresis. Datos para la vida. Bogotá: Imprenta Nacional de Colombia.

Instituto Nacional de Medicina Legal y Ciencias Forenses. (2013). Forensis. Datos para la vida. Bogotá: Imprenta Nacional de Colombia.

Instituto Nacional de Medicina Legal y Ciencias Forenses. (2014). Forensis. Datos para la vida. Bogotá: Imprenta Nacional de Colombia.

Instituto Nacional de Medicina Legal y Ciencias Forenses. (2015). Forensis Datos para la vida. Bogotá: Imprenta Nacional de Colombia. 
Restrepo et al - Caracterización del Suicidio en el departamento de Sucre.

Instituto Nacional de Medicina Legal y Ciencias Forenses. (2016). Forensis. Datos para la vida. Bogotá: Imprenta Nacional de Colombia.

Instituto Nacional de Medicina Legal y Ciencias Forenses. (2017). Forensis. Datos para la vida. Bogotá: Imprenta Nacional de Colombia.

Jimenez-Ornelas, R., \& Cardiel-Téllez, L. (s.f). El suicidio y su tendencia social en México: 1990 - 2011. Mexico.

Ley 1616. (31 de Mayo de 2013). Ley 1616. Ley 1616. Bogotá, Colombia.

Londoño, N., Muñiz, O., \& Correa, J. (2005). Salud Mental en Víctimas de la violencia armada en Bojayá (Chocó, Colombia). Revista Colombiana de Psiquiatría, 34(4), $493-505$.

Muñoz, E., \& Gutierrez, M. (2010). Factores de riesgo asociados al suicidio en Nariño (Colombia) estudio de casos y controles. Revista colombiana de psiquiatría, 39(2), $291-312$.

Muñoz, I., Bernal, J., Guarín, N., \& Arévalo, H. (2015). Caracterización de la demanda de servicios de salud mental en una aseguradora en salud en Colombia, 2012. Revista de psicología Universidad de Antioquía, 7(2), 63 - 80.

Muñoz, L., \& Sánchez, R. (2013). Caracterización de noticias sobre suicidio en medios impresos en Colombia. Revista de psiquiatría, 43(1), 12 - 18.

Ordoñez, I., Franco, S., \& Gozález, J. (2013). Caracterización sociodemográfica de los suidas adultos mayores de 60 años. Revista Colombiana de Psiquiátria, 43(1), 56 - 64.

Organización Mundial de la Salud (OMS). (2014). Preventing Suicide. Luxemburgo: OMS.

Palacio, J., Mandariaga, C., \& Sabatier, C. (2001). Estrés post-traumático en jóvenes desplazados por la violencia política en Colombia. Obtenido de Psiquiatría.Com: https://psiquiatria.com/estres-62/estres-post-traumatico-en-jovenes-desplazadospor-la-violencia-politica-en-colombia/

Peña, M., Ortiz, Y., \& Gutierrez, M. (2009). El suicidio en Nariño: Una mirada desde los observatorios del delito en cinco municipios del departamento. Pensamiento Psicológico, 6(13), 91 - 107.

Pérez, I., Téllez, D., Vélez, A., \& Ibáñez-Pinilla, M. (2012). Caracterizacion de factores asociados con comportamiento suicida en adolescentes estudiantes de octavo grado en tres colegios bogotanos. Revista Colombiana de Psiquiatría, 41(1), 26 - 47.

Ramírez-Giraldo, A., Hernández-Bustamante, O., Romero-Acosta, K., \& Porras-Mendoza, E. (2017). Trastornos de ansiedad y del estado de ánimo en personas víctimas del conflicto armado en Colombia: el caso de Chengue y de Libertad. Psicología desde el Caribe, 34(1), 30 - 41.

Redón-Quintero, E., \& Rodríguez-Gómez, R. (2016). Vivencias y experiencias de individuos con ideación e intento suicida. Revista colombiana de psiquiatría, 45(2), $92-100$. 
Rodriguez, M., Rodríguez, N., Gempeler, J., \& Garzón, F. (2013). Factores asociados con intento de suicidio y comportamientos de autolesión no suicida en pacientes con trastornos del comportamiento alimentario. Revista colombiana de psiquiatría, $43(1), 19-26$.

Rueda-Jaimes, E., Díaz, P., Rangel, A., Castro-Rueda, V., \& Camacho, P. (2011). Diferencias de género en pacientes con suicidabilidad. Revista Colombiana de Psiquiátria, 40(4), $637-646$.

Rueda-Jaimes, G., Martinez-Villalba, A., Castro-Rueda, V., \& Camacho, P. (2010). Suicidabilidad en adolescentes, una comparación con población adulta. Revista colombiana de psiquiatría, 39(4), 683 - 692.

RUV, R. U. (25 de Junio de 2018). Unidad de Víctimas. Obtenido de Unidad de Víctimas: https://www.unidadvictimas.gov.co/es

Sahagún-Navarro, M., Romero-Acosta, K., Millán-Beltrán, S., Arroyo-Arias, L, y RiveraJaraba, M. (2017). Violencia y vulnerabilidad: Estado del Arte sobre la vulnerabilidad de las Personas Mayores víctimas del conflicto armado colombiano. En C. Rivera, M. M. Carrillo (Coord.), Violencia y Desarrollo (pp. 185-206). Puebla, México: Instituto de Ciencias de Gobierno y Desarrollo Estratégico (ICGDE) de la Benemérita Universidad Autónoma de Puebla (BUAP). DOI: https://doi.org/10.21892/9786075254395

Spino, e., Kameg, K., Cline, T., Terhorst, L., \& Mitchell, A. (2016). Impact of social support on symptoms of depression and loneliness in survivors bereaved by suicide. Archiv of Psych., 30(5), 602 - 606.

Trout, Z., Hernández, E., \& Liu, R. (2017). Prospective prediction of first lifetime suicide attemps in a multi-site study of substance users. J of Psych Res, 84, 35 - 40.

Valencia, H., Campo-Cabal, G., Borrero, C., García, A., \& Patiño, M. (2011). Caracterización de la población con intento de suicidio en el Hospital Universitario del Valle. Revista Colombiana de Psiquiatría, 43(4), 619-636.

Vásquez, R., \& Quijano, M. (2013). Cuando el intento de suicidio es cosa de niños. Revista Colombiana de Psiquiátria, 43(1), 36 - 46.

Velázquez, J. (2013). Suicidio en el anciano. Revista colombiana de psiquiatría, 43(1), $80-84$.

World Health Organization. (17 de 07 de 2018). World Health Statistics data visualizations dashboard. Obtenido de World Health Statistics data visualizations dashboard: http://apps.who.int/gho/data/node.sdg.3-4-data?lang=en 